\title{
Megaureter e hidronefrose por ectopia ureteral extramural em cão
}

\author{
Yago Gabriel da Silva Barbosa ${ }^{1}$, Daniel Serafim de Andrade Rodrigues ${ }^{2}$, Eglesia \\ Rodrigues Leite ${ }^{2}$, Jefferson Rodrigues Araújo ${ }^{3}$, Charlys Rhands Coelho de Moura ${ }^{3}$, \\ Catarina Rafaela Alves da Silva ${ }^{4}$, Francisco Lima Silva ${ }^{5}$
}

${ }^{1}$ Médico veterinário, Residente em clínica e cirurgia de cães e gatos, Universidade Federal do Piauí, Teresina.
${ }^{2}$ Graduando em Medicina Veterinária, Universidade Federal do Piauí, Teresina.
${ }^{3}$ Médico veterinário, Residente em Diagnostico por Imagem, Universidade Federal do Piauí, Teresina.
${ }^{4}$ Médico veterinário, Residente Anestesiologia, Universidade Federal do Piauí, Teresina.
${ }^{5}$ Docente, Departamento de Clínica e Cirurgia Veterinária, Universidade Federal do Piauí, Teresina.
${ }^{*}$ Autor para correspondência, E-mail: y.ago.gabriel@hotmail.com

RESUMO. A ectopia ureteral é uma anomalia congênita do trato genito-urinário caracterizado por um distúrbio na inserção do ureter na vesícula urinária, sendo a causa mais comum de incontinência urinária em cães jovens, ocorrendo predominantemente em animais do sexo feminino. Anatomicamente é classificada como intramural ou extramural. $\mathrm{Na}$ intramural o ureterse anexa na superfície dorsal vesícula urinária, porém há falha ao desembocar no trígono vesical, enquanto que na extramural ocorre um desvio por completo da bexiga, podendo ocorrer em ambos os casos de forma unilateral ou bilateral. Os principais sinais clínicos incluem além da incontinência urinária, o eczema vulvar com hiperpigmentação, hipotricose e dermatite na região perivulvar, hematúria, piúria e cistites recorrentes. Objetivou neste artigo relatar o caso clínico de uma cadela da raça pinscher, 8 meses de idade, atendida no Hospital Veterinário da UFPI, diagnosticada e tratada para ectopia ureteral com megaureter e hidronefrose adquirida.

Palavras chave: Ectopia ureteral, megaureter, hidronefrose, cão.

\section{Megaureter and hydronephrosis by extramural ureteral ectopia in $\operatorname{dog}$}

\begin{abstract}
Ureteral ectopia is a congenital abnormality of the genito-urinary tract disorder characterized by the insertion of the ureter in the urinary bladder, the most common cause of urinary incontinence in young dogs, occurring predominantly in female animals. Anatomically it is classified as intramural or extramural. In the intramural ureter is attached to the dorsal surface of the urinary bladder, but has failed to culminate in the trigone, while the extramural there is a deviation in full bladder and can occur in both cases unilaterally or bilaterally. The main clinical signs include addition of urinary incontinence, vulvar eczema with hyperpigmentation, hypotrichosis and dermatitis in perivulvar region, hematuria, pyuria and recurrent cystitis. The objective of this article report the case of a bitch pinscher breed, 8 months old, attended at the Veterinary Hospital of UFPI, diagnosed and treated for ureteral ectopia with megaureter and acquired hydronephrosis.
\end{abstract}

Keywords: Ureteral ectopia, megaureter, hydronephrosis, dog.

\section{Introdução}

O Sistema urinário é composto pelos os rins, ureteres, bexiga e uretra. A função dos rins é produzir a urina que, por meio dos ureteres, chega à bexiga, onde é temporariamente armazenada. Os ureteres transportam urina dos rins para a bexiga e chegam a estes pelo hilo, onde se conectam a pelve renal ou estrutura equivalente, de acordo com a espécie animal.A inserção do seguimento final do ureter entre a musculatura vesical previne refluxo de urina para os ureteres quando aumenta a pressão intravesical (Feitosa, 2014).

A diferenciação anormal dos ductos mesonéfricos e metanéfricos durante a gênese 
embrionária proporciona uma condição anormal da relação vesico-ureteral conhecida com ectopia ureteral que é uma anomalia congênita do trato genito-urinário (Birchard \& Sherding, 2008).

Anatomicamente a ectopia ureteral pode ser classificada como intramural e extramural. O tipo intramural se anexa na superfície dorsal ou dorsolateral da vesícula urinária, mas falha ao desembocar no trígono vesical, o que passa a ocorrer no colo da vesícula urinária, uretra ou vagina; já o extramural tem um desvio por completo da bexiga podendo ocorrer uni ou bilateral, ocorrendo com mais frequência no colo vesical, bexiga, uretra proximal, medial ou distal, vagina ou útero (McLoughlin \& Chew, 2000).

Os ureteres ectópicos são a causa mais comum de incontinência urinária em cães jovens e acomete predominantemente fêmeas, sendo o sinal clínico mais evidente em todas as espécies (Canola et al., 2006). Outros sinais comuns incluem o eczema vulvar com hiperpigmentação, hipotricose e dermatite na região perivulvar, hematúria, piúria e cistites recorrentes (McLoughlin \& Chew, 2000, Silva et al., 2012) podendo ainda muitas vezes associa-se ao hidroureter e à hidronefrose (Oliveira et al., 2013).

A correção cirúrgica é o tratamento de escolha, sendo que deve ser realizada o mais rápido possível, para evitar possíveis infecções urinárias como a infecção ascendente e obstrução uretral e outras complicações associadas ao comprometimento das funções urinárias ( al., 2012).

O principal tratamento cirúrgico do ureter ectópico inclui a ureteronefrectomia para casos em que o rim se encontra danificado ou não funcional, ureteroneocistotomia para reimplantação do ureter ectópico extramural dentro da bexiga e a neoureterostomia para criação de uma nova abertura para o ureter ectópico intramural dentro da bexiga (Taney et al., 2003).

Objetivou-se neste artigo relatar o caso clínico de uma cadela da raça pinscher, 8 meses de idade atendida no Hospital Veterinário da UFPI, diagnosticada e tratada para ureter ectópico com hidronefrose adquirida.

\section{Descrição do caso}

Foi atendida no HVU da UFPI, uma cadela da raça pinscher, 8 meses de idade, não castrada e com peso de 2,2 kg. O tutor queixava-se de uma intermitente incontinência urinária desde $o$ nascimento, sendo que a mesma já havia passado por outros tratamentos para o mesmo problema, porém a etiologia ainda não havia sido diagnosticada. Durante o exame físico, o animal apresentava-se consciente e alerta, mucosa ocular e oral normocoradas, temperatura retal (TR) de $38,2^{\circ} \mathrm{C}$, Frequência Cardíaca (FC) de 142 bpm e frequência respiratória (FR) de $45 \mathrm{mpm}$.A Vulva apresentava-se edemaciada com presença de algumas pústulas na região perivulvar.

Foram solicitados os exames complementares, hemograma, bioquímico (uréia, creatinina, ALP/TGP, fosfatase alcalina, proteína total, albumina e globulina), urinálise, ultra-som abdominal e urografia excretora. No hemograma foi observado discreta macrocitose e policromasia, com presença de macroplaquetas e hemácias microcíticas e hipocrômicas. No exame bioquímico foi observado elevação dos níveis de Uréia (87,4mg/dL), ALT/TGP (89U/L), Fosfatase alcalina 169,3 (U/L) e uma diminuição nos níveis de Proteína total $(5,2 \mathrm{~g} / \mathrm{dL})$ e Globulina $(2,5 \mathrm{~g} / \mathrm{dL}) . \mathrm{Na}$ urinálise não foram observado alterações.

$\mathrm{Na}$ ultrassonografia abdominal foi observado no rim direito uma acentuada dilatação da pelve renal com presença de líquido (Hidronefrose) e aparente dilatação do ureter em toda a sua extensão com inserção ectópica na uretra. $\mathrm{Na}$ urografia excretora foi possível visualizar a estrutura do megaureter e o local o de sua inserção, sendo diagnosticado como um caso de ureter ectópico direito do tipo extramural, desembocando na porção cranial da uretra (Figura 1a e 1b).

Após a avaliação física e dos exames solicitados, optou-se pelo tratamento cirúrgico. A técnica cirúrgica de escolha foi a ureteronefrectomia unilateral modificada, que consiste na remoção do rim patológico e do ureter correspondente (Figura 2). O rim removido apresentava áreas hemorrágicas, $3,4 \mathrm{~cm}$ de diâmetro e pelve renal bastante dilatada (Figura. $3)$.

No pós-operatório imediato foi realizado o controle da dor com a utilização de morfina $(0,5 \mathrm{mgq} / \mathrm{kg})$ e dexametasona $(0,5 \mathrm{mg} / \mathrm{kg})$ por 2 dias e antibioticoterapia a base de cefalexina $(20 \mathrm{mg} / \mathrm{kg})$. Após os dois dias, com o animal estabilizado optou-se pela alta médica, com mudança na terapêutica do animal onde este 
passou a fazer uso de tramadol $(2 \mathrm{mg} / \mathrm{kg})$ associado a dipirona sódica $20 \mathrm{mg} / \mathrm{kg}$, e carprofeno $(4,4 \mathrm{mg} / \mathrm{kg})$ por 6 dias, mantendo o antibiótico por 10 dias. O tutor foi instruído a fornecer dieta a base de ração renal e retornar para reavaliação após 21 dias. Após 21 dias, o animal retornou ao hospital veterinário para revisão clínica e laboratorial. O paciente demonstrou bem-estar geral, ausência de alterações nos exames complementares e cicatrização total da ferida cirúrgica (figura 4), sem sinais clínicos compatíveis com alterações no trato urinário, comprovando a eficiência da ureteronefrectomia para o tratamento da paciente com incontinência urinaria por ureter ectópico com megaureter e hidronefrose recorrente.
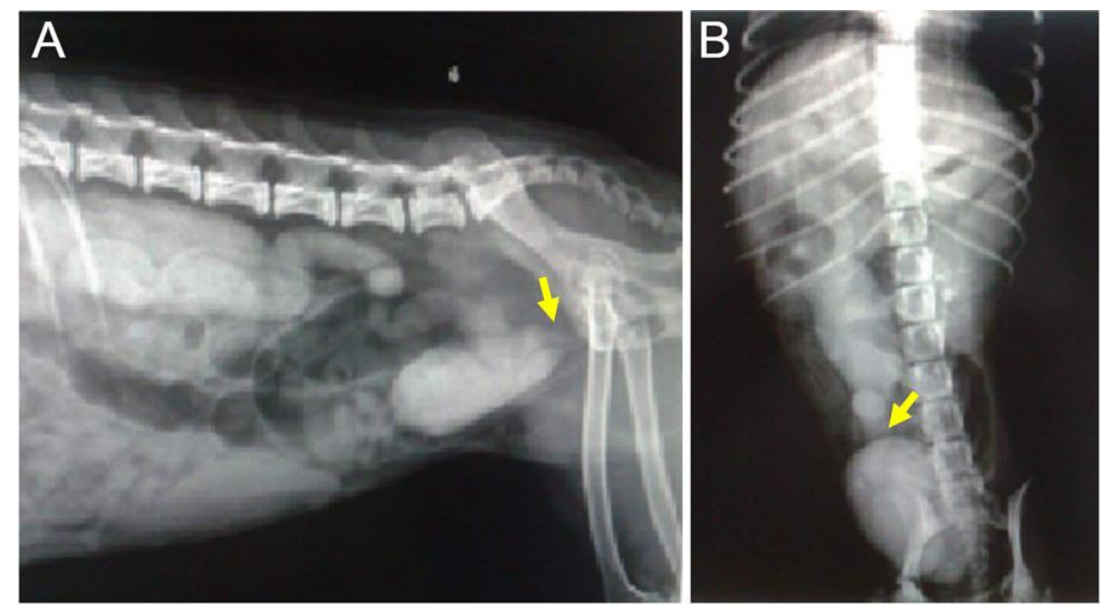

Figura 1. Cadela, Pinscher, 8 meses, apresentando Ureter ectópico com megaureter recorrente (seta). Radiografia, após exame de urografia excretora. A-Decúbito látero-lateral. B-Decúbito dorsal.

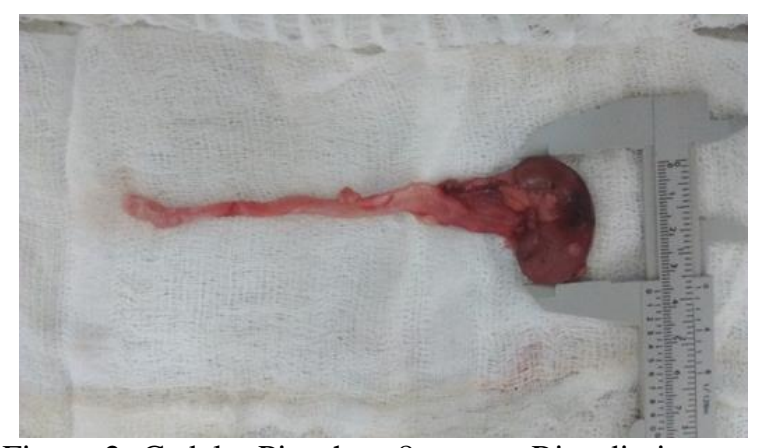

Figura 2. Cadela, Pinscher, 8 meses. Rim direito com hidronefrose medindo $3,4 \mathrm{~cm}$.

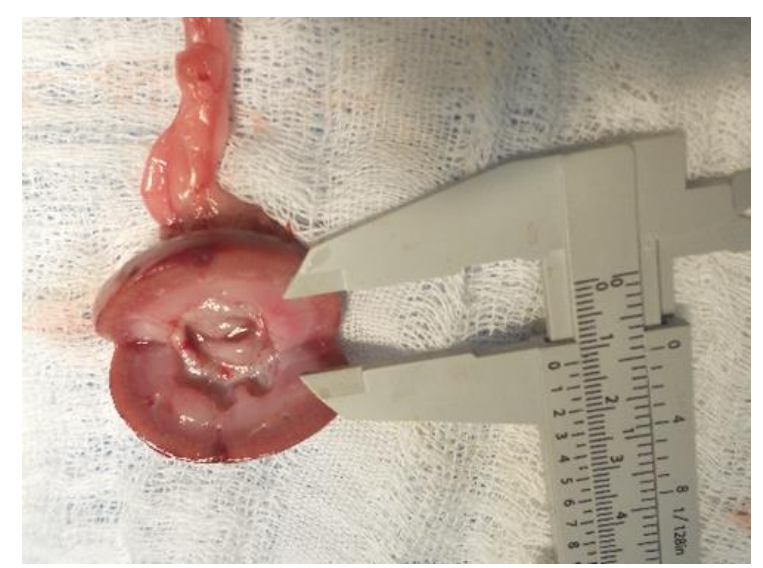

Figura 3. Cadela. Pinscher, 8 meses. Aspectos macroscópicos do rim direito com hidronefrose e pelve renal dilatada.

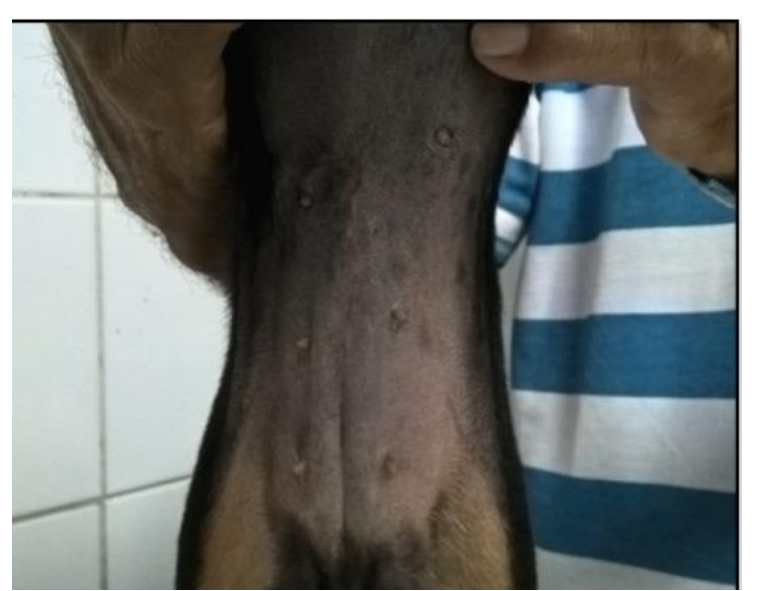

Figura 4: Cadela, Pinscher, 8 meses, atendida no Hospital Veterinário Universitário, 21 dias após a realização de ureteronefrectomia.

\section{Discussão}

No presente relato as alterações anatômicas, fisiológicas, histórico e sinais clínico corroboram com os achados descrito na literatura (Costa Neto et al., 2013, Bianchi et al., 2013), sendo evidenciado que há uma maior correlação com animais do sexo feminino e filhotes apresentando incontinência urinária desde o nascimento, presença de pústulas na região perivulvar e vaginite. Assim como relatado por Silva et al. (2012), Costa Neto et al. (2013) e Oliveira et al. 
(2013) observou-se hidronefrose, uma dilatação da pélvis e dos cálices reanis, por obstrução do fluxo fisiológico da urina, em consequência da qual se estabelece atrofia por compressão do parênquima renal, alteração esta que podem atingir graus extremos com estabelecimento de uremias que podem levam o animal à morte.

No presente caso, ocorreu concomitante à ectopia ureteral e hidronefrose o desenvolvimento de megaureter; como relatado por Oliveira et al. (2013) porém na maioria dos relatos consultados tenha se observado uma maior prevalência de hidroureter (Silva et al., 2012, Bianchi et al., 2013). Segundo Kealy et al. (2005), os ureteres não podem ser visualizados em radiografias simples, portanto foi utilizado meios contrastados para alcançar o diagnóstico.

$\mathrm{Na}$ ultrassonografia foi possível observar alteração renal com constatação da dilatação da pelve renal e ureter com aparente implantação ectópica na bexiga urinária. Todavia, o diagnóstico definitivo do ureter ectópico foi obtido pela urografia excretora, assim como o recomendado por McLoughlin \& Chew (2000) e Oliveira et al. (2013), permitindo pela imagem, diagnosticar se a ectopia ureteral é uni ou bilateral, tamanho, localização, morfologia e locais de implantações dos ureteres. Na paciente, a inserção do ureter estava localizado na porção cranial da uretra, corroborando com os achados de Bianchi et al. (2013), Costa Neto et al. (2013) e Oliveira et al. (2013).

Apesar de o rim direito apresentava filtração, evidenciada pela urografia excretora sabe-se que bastam 5\% da função renal preservada para se observar radiograficamente a excreção do meio de contraste (Larson, 2009). Assim foi adotado o tratamento cirúrgico, com a realização da ureteronefrectomia; pois o mesmo já apresentava hidronefrose, bem como megaureter e ectopia ureteral direita assim como o preconizado por Rawlings (2005) e Costa Neto et al. (2013) quando o rim contra lateral encontrava-se em funcionamento normal.

\section{Conclusão}

O clínico veterinário deve incluir a ectopia ureteral como suspeita clínica sempre que cães jovens apresentarem sintomas de incontinência urinária desde o nascimento, principalmente em animais do sexo feminino, apresentando eczema vulvar com hiperpigmentação, hipotricose e dermatite na região perivulvar, hematúria, piúria ou cistites recorrentes. O diagnostico definitivo é obtido através urografia excretora e tratamento é realizado por meio cirúrgico.

\section{Referências bibliográficas}

Bianchi, S. P., Gouvêa, A. S., Macedo, A. S., Valente, F. S., Ledur, G. R., Alievi, M. M. \& Contesini, E. A. (2013). Ureter ectópico extramural em cadela. Acta Scientiae Veterinariae, 41, 1-4.

Birchard, S. J. \& Sherding, R. G. (2008). Manual Saunders: clínica de pequenos animais, São Paulo.

Canola, J. C., Lacreta JR, A. C. C., Sanches, R. C. \& Maniscalco, C. L. (2006). Incontinência urinária em cadela associada com ureter e ureterocele ectópicos. Revista Nosso Clinico, 9, 36-42.

Costa Neto, J. M., Silva, A. E., Filho, E. F. M., Ribeiro, L. G. R., Gama, R. O. G., Penha, E. M., Toríbio, J. M. d. M. L. \& Júnior, D. C. G. (2013). Ectopia ureteral em cães: relato de dois casos. Arquivos de Ciências Veterinárias e Zoologia, 14, 151-156.

Feitosa, F. L. F. (2014). Semiologia veterinária: A arte do diagnóstico. Grupo Gen-Editora Roca Ltda., São Paulo.

Kealy, J. K., McAllister, H. \& Graham, J. P. (2005). Radiologia e Ultrassonografia do Cão e do Gato. Manole, São Paulo.

Larson, M. M. (2009). The kidneys and ureters. Manual of canine and feline abdominal imaging, 1, 185-204.

McLoughlin, M. A. \& Chew, D. J. (2000). Diagnosis and surgical management of ectopic ureters. Clinical Techniques in Small Animal Practice, 15, 17-24.

Oliveira, A. L. A. (2012). Técnicas cirúrgicas de pequenos animais. Rio de Janeiro: Elsevier. 1 ed. 480 p.,.

Oliveira, D. M. M. C., Reusing, M. S. O. \& Silva, D. M. (2013). Ectopia ureteral bilateral em cadelas: diagnóstico, tratamento cirúrgico e evolução clínica. Acta Scientiae Veterinariae, 41, 1- 4.

Rawlings, C. A. (2005). Ureter. In: Bojrab, M. J. (ed.) Técnicas atuais em cirurgia de pequenos animais. Roca, São Paulo. 
Silva, M. N., Larangeira, D. F., Penha, E. M., Oriá, A. P., Costa Neto, J. M. \& BarrouinMelo, S. M. (2012). Ectopia ureteral unilateral congênita em uma cadela Teckel Dachshund com pelagem arlequim: relato de caso. Arquivo Brasileiro de Medicina Veterinaria e Zootecnia, 64, 1504-1510.

Taney, K. G., Moore, K. W., Carro, T. \& Spencer, C. (2003). Bilateral ectopic ureters in a male dog with unilateral renal agenesis. Journal of the American Veterinary Medical Association, 223, 817-820.
Recebido em Março 31, 2016

Aceito em Maio 5, 2016

License information: This is an open-access article distributed under the terms of the Creative Commons Attribution License, which permits unrestricted use, distribution, and reproduction in any medium, provided the original work is properly cited. 